\title{
DIOPHANTINE APPROXIMATION WITH ARITHMETIC FUNCTIONS, I
}

\author{
EMRE ALKAN, KEVIN FORD AND ALEXANDRU ZAHARESCU
}

\begin{abstract}
We prove a strong simultaneous Diophantine approximation theorem for values of additive and multiplicative functions provided that the functions have certain regularity on the primes.
\end{abstract}

\section{IntroduCtion}

There is a rich literature on problems of approximating real numbers by rational numbers with multiplicative restrictions on the denominator of the rational number, e.g. [1], [2], [10] and the references therein. We are concerned here with approximating real numbers by values of additive and multiplicative functions. One of the classical results in this area is the 1928 theorem of Schoenberg [16], which states that $\phi(n) / n$ has a continuous distribution function, that is,

$$
F(z)=\lim _{x \rightarrow \infty} \frac{1}{x}|\{n \leq x: \phi(n) / n \leq z\}|
$$

exists for every real $z$, and $F(z)$ is continuous. Here $\phi$ is Euler's totient function. In particular, $\phi(n) / n$ is dense in $[0,1]$, or equivalently, the additive function $\log \phi(n) / n$ is dense in $(-\infty, 0]$ (in general, $f(n)$ is additive if and only if $e^{f(n)}$ is multiplicative). Erdős and Wintner [9] later determined precisely which real additive functions have continuous distribution functions. These include $\log \phi(n) / n$ and its close cousin $\log \sigma(n) / n$, where $\sigma(n)$ is the sum of the divisors of $n$. A stronger approximation theorem was proved by Wolke [17]. Let $\Gamma$ denote the infimum of numbers $\gamma$ so that for large $x$, there is a prime in $\left(x-x^{\gamma}, x\right]$. Wolke proved that for any real $\beta \geq 1$ and any $c<1-\Gamma$, there are infinitely many integers $n$ with $\left|\frac{\sigma(n)}{n}-\beta\right|<n^{-c}$. It is conjectured that $\Gamma=0$, however we only know that $\Gamma \leq 0.525$ [3].

In the 1950's, several papers appeared concerning the distribution of values of $\phi(n)$ and the sum of divisors function $\sigma(n)$ at consecutive integers. A major unsolved problem is whether, for fixed $k \neq 0$, the equations $\phi(x+k)=\phi(x)$ or

Date: May 10, 2008.

2000 Mathematics Subject Classification. 11N64, 11N36, 11K60.

Key words and phrases: Diophantine approximation, additive functions, multiplicative functions.

Second author supported in part by National Science Foundation Grant DMS-0555367. Third author supported in part by National Science Foundation Grant DMS-0456615. 
$\sigma(x+k)=\sigma(x)$ have infinitely many solutions. For the latest work on the problem for $\phi$, see [11]. Schinzel [15] proved that for any $h \geq 1, \varepsilon>0$ and positive real numbers $\alpha_{1}, \cdots, \alpha_{h}$, the system of simultaneous inequalities

$$
\left|\frac{\phi(n+i)}{\phi(n+i-1)}-\alpha_{i}\right|<\varepsilon \quad(1 \leq i \leq h)
$$

has infinitely many solutions. Six years later Schinzel teamed with Erdős [8] to show that (1.1) holds for a positive proportion of integers $n$, and to generalize this result to a wide class of additive and multiplicative functions.

It is interesting to ask how fast $\varepsilon=\varepsilon(n)$ can tend to zero as a function of $n$ in (1.1). In particular, Erdös [7] posed the problem to show that for some $c<1$, the inequalities

$$
|\phi(n+1)-\phi(n)|<n^{c} \quad \text { and } \quad|\sigma(n+1)-\sigma(n)|<n^{c}
$$

each have infinitely many solutions.

Our first results solve Erdős' problem and generalize the aforementioned theorems of Schinzel and Wolke. In particular, we may replace $\varepsilon$ on the right side of (1.1) with $n^{-c_{h}}$ for some positive $c_{h}$. As in [8], we state our results for a wide class of additive functions. For any $\delta>0$ and $\lambda>0$ we denote by $\mathcal{F}_{\delta, \lambda}$ the set of additive functions $f: \mathbb{N} \rightarrow \mathbb{R}$ with the following properties:

(a) We have

$$
\sum_{\substack{p \text { prime } \\ f(p)>0}} f(p)=\infty .
$$

(b) There exists a constant $C(f)>0$ depending on $f$ such that

$$
\left|f\left(p^{v}\right)\right| \leq \frac{C(f)}{p^{\delta}}
$$

for any prime number $p$ and $v \geq 1$.

(c) There exists $t_{0}(f)>0$ depending on $f$ such that for any $0<t \leq t_{0}(f)$ there is a prime number $p$ satisfying

$$
t-t^{1+\lambda} \leq f(p) \leq t
$$

We remark that (a) and (b) imply that $\delta \leq 1$. Also, (b) and (c) together imply that $\delta \lambda<1$. To see this, let $\varepsilon>0$ and $S=|\{p: f(p)>\varepsilon\}|$. By (b),

$$
S \leq\left|\left\{p: C(f) / p^{\delta}>\varepsilon\right\}\right|=o\left(\varepsilon^{-1 / \delta}\right) \quad\left(\varepsilon \rightarrow 0^{+}\right) .
$$

On the other hand (cf. (2.2) below), the interval $\left[\varepsilon, t_{0}(f)\right]$ contains $\gg \varepsilon^{-\lambda}$ disjoint intervals of the form $\left(t-t^{1+\lambda}, t\right]$. Hence $S \gg \varepsilon^{-\lambda}$. Finally, if (c) holds with $\lambda>1$ then (a) follows.

Theorem 1. Fix an integer $k \geq 1$ and real numbers $0<\delta \leq 1,0<\lambda<1 / \delta$. Let $f_{1}, \ldots, f_{k}$ be functions in $\mathcal{F}_{\delta, \lambda}$, and let $A=1$ if all $f_{i}$ are identical, and $A=2$ 
otherwise. Suppose $a_{1}, \ldots, a_{k}$ are positive integers, $b_{1}, \ldots, b_{k}$ are nonzero integers and

$$
a_{i} b_{j} \neq a_{j} b_{i} \quad(1 \leq i<j \leq k) .
$$

If $\alpha_{i}>f_{i}\left(b_{i}\right)$ for $1 \leq i \leq k$ and

$$
0<c< \begin{cases}\delta \lambda & \text { if } k=1 \text { and } a_{1} \in\{1,2\} \\ \frac{\delta \lambda}{A k+\lambda \beta_{k}} & \text { otherwise, }\end{cases}
$$

then there are infinitely many positive integers $m$ satisfying

$$
\left|f_{i}\left(a_{i} m+b_{i}\right)-\alpha_{i}\right|<\frac{1}{m^{c}} \quad(1 \leq i \leq k) .
$$

Here $\beta_{k}$ is an admissible value of a "sieve limit for a sieve of dimension $k$ ", which will be described below (Theorem DHR). In particular, $\beta_{1}=2, \beta_{2}<4.2665$ and $\beta_{k}=O(k)$.

Theorem 2. Fix an integer $k \geq 1$ and real numbers $0<\delta \leq 1,0<\lambda<1 / \delta$. Let $f_{0}, \ldots, f_{k}$ be functions in $\mathcal{F}_{\delta, \lambda}$, with $A=1$ if $f_{1}, \ldots, f_{k}$ are identical and $A=2$ otherwise. Suppose $a_{0}, a_{1}, \ldots, a_{k}$ are positive integers, $b_{1}, \ldots, b_{k}$ are integers and (1.2) is satisfied. If $\zeta_{1}, \ldots, \zeta_{k}$ are arbitrary real numbers, and

$$
0<c< \begin{cases}\frac{\delta \lambda}{1+4 \lambda} & \text { if } k=1 \\ \frac{\delta \lambda}{A k+\lambda \beta_{k+1}} & \text { if } k \geq 2,\end{cases}
$$

then there are infinitely many positive integers $m$ satisfying

$$
\left|f_{i}\left(a_{i} m+b_{i}\right)-f_{i-1}\left(a_{i-1} m+b_{i-1}\right)-\zeta_{i}\right|<\frac{1}{m^{c}} \quad(1 \leq i \leq k) .
$$

Remarks. Theorem 1 implies immediately the conclusion of Theorem 2 in the range

$$
0<c<\frac{\delta \lambda}{A(k+1)+\lambda \beta_{k+1}}
$$

by choosing $\alpha_{0}, \ldots, a_{k}$ large and satisfying $\alpha_{i}-\alpha_{i-1}=\zeta_{i}$ for each $i$. Larger values of $c$ are possible by making a more judicious choice of $\alpha_{0}$.

If we assume the Elliott-Halberstam conjecture on the distribution of primes in arithmetic progressions, then the conclusion of Theorem 2 for $k=1$ holds for

$$
0<c<\frac{\delta \lambda}{1+2 \lambda}
$$

Corollaries. We may apply Theorems 1 and 2 to the functions $f(n)=\log (n / \phi(n))$ and $f(n)=\log (\sigma(n) / n)$. Each of these satisfies $f(p)=\frac{1}{p}+O\left(\frac{1}{p^{2}}\right)$. It follows that $f \in \mathcal{F}_{1, \lambda}$ for any $\lambda<1-\Gamma$. Applying Theorem 1 with $k=1, a_{1}=1$ 
and $f_{1}(n)=\log (\sigma(n) / n)$ recovers Wolke's result. Applying Theorem 2 with $f_{i}(n)=\log (n / \phi(n))$ shows that one may take $\varepsilon=n^{-c_{h}}$ in (1.1) provided that

$$
c_{h}<\frac{1-\Gamma}{h+(1-\Gamma) \beta_{h+1}} .
$$

We also have an answer to Erdös' question, by applying Theorem 2 with $k=1$, $\zeta_{1}=0, a_{0}=a_{1}=b_{1}=1$ and $b_{0}=0$. For any $c<\frac{1-\Gamma}{5-4 \Gamma}$, the inequalities

$$
|\phi(n+1)-\phi(n)|<n^{1-c}, \quad|\sigma(n+1)-\sigma(n)|<n^{1-c}
$$

each have an infinite number of solutions. In addition, for any nonzero $a, c<\frac{1-\Gamma}{5-4 \Gamma}$ and for any real $\zeta$, the inequality

$$
|n / \phi(n+a)-\sigma(n) / n-\zeta|<n^{-c}
$$

has infinitely many solutions.

The methods used to prove Theorems 1 and 2 also yield similar results for the simultaneous approximation of $f_{i}\left(g_{i}(n)\right)$ where $g_{i}(n)$ are polynomials, provided that (a) and (c) above are suitably strengthened. Rather than aim for fullest generality, we illustrate what is possible with two special cases.

Theorem 3. Suppose $h(n)=\phi(n)$ or $h(n)=\sigma(n)$. Let $\Gamma^{\prime}$ be the infimum of numbers $g$ so that if $x$ is large, there is a prime $p \equiv 1(\bmod 4)$ with $x-x^{g}<p \leq x$. For any real $\zeta$ and any

$$
c<\frac{1-\Gamma^{\prime}}{1+\left(1-\Gamma^{\prime}\right) \beta_{2}}
$$

the inequality

$$
\left|h\left(n^{2}+1\right)-h\left(n^{2}+2\right)-\zeta\right|<n^{2-c}
$$

has infinitely many solutions.

A sketch of the proof of Theorem 3 will appear in section 4 , together with a discussion of how to deal with more general $f_{i}$ and $g_{i}$. Likely the methods in [3] can be used to prove $\Gamma^{\prime} \leq 0.525$, but the best result available in the literature is $\Gamma^{\prime} \leq 0.53$ (by considering the polynomial $Q(x, y)=x^{2}+y^{2}$ in [13]).

In [14], a similar Diophantine approximation problem is considered for consecutive values of the kernel function $k(n)=\prod_{p \mid n} p$. Our methods do not apply, since $f(p)=0$ for $f(n)=\log (n / k(n))$. Luca and Shparlinski [14] show that for any vector $\left(\alpha_{1}, \ldots, \alpha_{k}\right)$ of positive real numbers, there are infinitely many $n$ for which

$$
\left|\frac{k(n+i-1)}{k(n+i)}-\alpha_{i}\right|<\frac{1}{n^{1 / 41 k^{3}}} \quad(1 \leq i \leq k-1) .
$$

In a sequel paper, we will consider Diophantine approximation problems for coefficients of modular forms. A example of one of our results is that for any real 
$\beta$, there is a constant $C_{\beta}$ so that for infinitely many $n$,

$$
\left|\frac{\tau(n)}{n^{11 / 2}}-\beta\right| \leq \frac{C_{\beta}}{\log n},
$$

where $\tau(n)$ is Ramanujan's function, the $n$th coefficient of $q \prod_{m=1}^{\infty}\left(1-q^{m}\right)^{24}$.

\section{Preliminaries for Theorems 1,2 And 3}

Lemma 1. Let $0<\delta \leq 1,0<\lambda<1 / \delta, f_{1}, \cdots, f_{k} \in \mathcal{F}_{\delta, \lambda}, 0<\xi<\lambda / A$, and $K \geq 1$. For sufficiently small positive $v_{0}$, there are disjoint sets $\mathcal{P}_{1}, \ldots, \mathcal{P}_{k}$ of primes greater than $K$ with the following properties. (i) Let $v_{j+1}=v_{j}-v_{j}^{1+\xi}$ for $j \geq 0$. For each $j \geq 0$ and $1 \leq i \leq k, \mathcal{P}_{i}$ contains exactly one prime $p$ with $f_{i}(p) \in\left(v_{j+1}, v_{j}\right]$. (ii) Let $\mathcal{P}_{0}$ be the set of primes larger than $K$ which do not lie in any set $\mathcal{P}_{i}$. Then

$$
\sum_{\substack{p \in \mathcal{P}_{0} \\ f_{i}(p)>0}} f_{i}(p)=\infty \quad(1 \leq i \leq k) .
$$

Proof. It is straightforward to show that for any $v_{0}$,

$$
v_{j} \sim \xi^{-1 / \xi} j^{-1 / \xi} \quad(j \rightarrow \infty)
$$

One method of proof is to compare $v_{j}$ to $y(j)$, where $y$ satisfies the differential equation $y^{\prime}=-y^{1+\xi}$. We will take $v_{0}$ satisfying

$$
\begin{aligned}
& v_{0}<\min _{1 \leq i \leq k} t_{0}\left(f_{i}\right), \\
& v_{0}<\min _{1 \leq i \leq k} \min \left\{f_{i}(p): p \leq K \text { and } f_{i}(p)>0\right\} .
\end{aligned}
$$

If $f_{1}, \ldots, f_{k}$ are identical, we also assume that

$$
v_{0}^{\xi-\lambda} \geq 2 k
$$

Since $\xi<\lambda, v_{0}$ satisfies (2.3), (2.4), and (2.5) if $v_{0}$ is small enough. If $f_{1}, \ldots, f_{k}$ are not identical, then $\xi<\frac{1}{2} \lambda$. By (2.2), if $v_{0}$ is small enough then

$$
v_{j}^{\xi-\lambda} \geq 2 k^{2}(j+1) \quad(j \geq 0) .
$$

Next, we construct the sets $\mathcal{P}_{i}$. First assume $f_{1}, \cdots, f_{k}$ are identical. By (2.5), for each $j \geq 0$ the interval $\left(v_{j+1}, v_{j}\right]$ contains at least $2 k$ disjoint intervals of the form $\left(v-v^{1+\lambda}, v\right]$. By (c) and (2.3), each such interval contains a value of $f_{1}(p)$ for some prime $p$. Label these $2 k$ primes $p_{i, j}, p_{i, j}^{\prime}$ for $1 \leq i \leq k$.

Assume that $f_{1}, \cdots, f_{k}$ are not all identical. Fix $j \geq 0$ and assume that we have chosen distinct primes $p_{i, h}, p_{i, h}^{\prime}$ such that $f_{i}\left(p_{i, h}\right), f_{i}\left(p_{i, h}^{\prime}\right) \in\left(v_{h+1}, v_{h}\right]$ for $1 \leq i \leq k$, $0 \leq h \leq j-1$. By $(2.6),\left(v_{j+1}, v_{j}\right]$ contains at least $2 k^{2}(j+1)$ intervals of the form $\left(v-v^{1+\lambda}, v\right]$. At most $2 k(k-1) j$ of these intervals contain a number of the form $f_{i^{\prime}}\left(p_{i, h}\right)$ or $f_{i^{\prime}}\left(p_{i, h}^{\prime}\right)$ for $1 \leq i \leq k, 1 \leq i^{\prime} \leq k, i \neq i^{\prime}, 0 \leq h \leq j-1$. Let $T$ denote the set of remaining intervals, so that $|T|>2 k^{2}$. Take two intervals $I_{1}, I_{1}^{\prime} \in T$. 
By (2.3) and (c), there are primes $p_{1, j}, p_{1, j}^{\prime}$ with $f_{1}\left(p_{1, j}\right) \in I_{1}$ and $f_{1}\left(p_{1, j}^{\prime}\right) \in I_{1}^{\prime}$. Take 4 intervals in $T \backslash\left\{I_{1}, I_{1}^{\prime}\right\}$. By (2.3) and (c), there are two of these intervals $I_{2}$ and $I_{2}^{\prime}$ and primes $p_{2, j}, p_{2, j}^{\prime}$ different from $p_{1, j}$ and $p_{1, j}^{\prime}$ so that $f_{2}\left(p_{2, j}\right) \in I_{2}$ and $f_{2}\left(p_{2, j}^{\prime}\right) \in I_{2}^{\prime}$. Continuing this process, since $|T| \geq 2+4+\cdots+2 k$, we can find $2 k$ distinct intervals $I_{1}, I_{1}^{\prime}, \ldots, I_{k}, I_{k}^{\prime} \in T$ and $2 k$ distinct primes $p_{1, j}, p_{1, j}^{\prime}, \ldots, p_{k, j}, p_{k, j}^{\prime}$, different from the previously chosen primes $p_{i, h}, p_{i, h}^{\prime}(1 \leq i \leq k, h<j)$ with $f_{i}\left(p_{i, k}\right) \in I_{i}$ and $f_{i}\left(p_{i, k}^{\prime}\right) \in I_{i}^{\prime}$ for $1 \leq i \leq k$.

In either case, for $1 \leq i \leq k$ let

$$
\mathcal{P}_{i}=\left\{p_{i, 0}, p_{i, 1}, \ldots\right\}
$$

By (2.4), all primes $p_{i, j}, p_{i, j}^{\prime}$ are larger than $k$. Hence, the sets $\mathcal{P}_{1}, \cdots, \mathcal{P}_{k}$ satisfy condition (i) of the lemma. If $\sum_{j \geq 0} v_{j}$ converges (that is, $\xi<1$ ), then for each $i$,

$$
\sum_{p \in \mathcal{P}_{i}} f_{i}(p)<\infty
$$

and hence by (a), (2.1) holds. Next assume $\sum_{j \geq 0} v_{j}$ diverges. For each $i$ and every $j \geq 0, p_{i, j}^{\prime} \in \mathcal{P}_{0}$ and $f_{i}\left(p_{i, j}^{\prime}\right) \in\left(v_{j+1}, v_{j}\right]$. Hence $(2.1)$ holds in this case as well.

Lemma 2. Let $0<\delta \leq 1,0<\lambda<1 / \delta, f_{1}, \cdots, f_{k} \in \mathcal{F}_{\delta, \lambda}$, and $0<\xi<\lambda / A$. Also assume that $K \geq 1$ and that $\gamma_{1}, \ldots, \gamma_{k}$ are positive real numbers. For sufficiently small $v_{0}$ and for

$$
0<\eta<\min \left(v_{0}, 6^{-1 / \xi}, \gamma_{1}, \ldots, \gamma_{k}\right)
$$

there are sequences $\left\{n_{i, j}\right\}, 1 \leq i \leq k, j=0,1,2, \ldots$, such that

(i) $n_{i, j} \mid n_{i, j+1}$ for each $1 \leq i \leq k$ and $j \geq 0$;

(ii) For each $j$, the numbers $n_{1, j}, n_{2, j}, \ldots, n_{k, j}$ are pairwise relatively prime and divisible by no prime $\leq K$;

(iii) $\left|f_{i}\left(n_{i, j}\right)-\gamma_{i}\right| \leq 3^{j} \eta^{(1+\xi)^{j}}$ for $1 \leq i \leq k, j \geq 0$;

(iv) we have

$$
n_{i, j} \leq \frac{\left(2 C\left(f_{i}\right)\right)^{\frac{j}{\delta}} n_{i, 0}}{(\eta / 2)^{\frac{(1+\xi)^{j}}{\delta \xi}}} \quad(1 \leq i \leq k, j \geq 0) .
$$

Assume (2.7) is satisfied, and let $\mathcal{P}_{0}, \mathcal{P}_{1}, \ldots, \mathcal{P}_{k}, v_{1}, \ldots$ be as in Lemma 1 . By (2.1) and (b), there are prime numbers $q_{1}, q_{2}, \ldots, q_{r}$ in $\mathcal{P}_{0}$ such that

$$
\gamma_{1}-\eta<\sum_{s=1}^{r} f_{1}\left(q_{s}\right)<\gamma_{1}-\frac{\eta}{2}
$$

We take

$$
n_{1,0}=\prod_{s=1}^{r} q_{s}
$$


so that $\gamma_{1}-\eta<f_{1}\left(n_{1,0}\right)<\gamma_{1}-\frac{\eta}{2}$. In this way we may successively construct $n_{i, 0}$ for $2 \leq i \leq k$. Assume that we have already constructed $n_{1,0}, n_{2,0}, \ldots, n_{i-1,0}$ with prime divisors in $\mathcal{P}_{0}$. If $\mathcal{B}_{i}$ is the set of all prime divisors of $n_{1,0}, n_{2,0}, \ldots, n_{i-1,0}$, then by $(2.1)$,

$$
\sum_{p \in \mathcal{P}_{0} \backslash \mathcal{B}_{i}} f_{i}(p)=\infty
$$

Therefore, by (b) we may choose $n_{i, 0}$ with all prime divisors in $\mathcal{P}_{0} \backslash \mathcal{B}_{i}$ and with $\gamma_{i}-\eta<f_{i}\left(n_{i, 0}\right)<\gamma_{i}-\frac{\eta}{2}$.

Next we construct $n_{1, j}$ for $j \geq 1$. Put $\tau_{1,0}=\gamma_{1}-f_{1}\left(n_{1,0}\right) \in\left(\frac{\eta}{2}, \eta\right)$ and recursively define $n_{1, j+1}=n_{1, j} p_{1, j+1}$ for $j \geq 0$ where $p_{1,1}, p_{1,2}, \ldots$ are prime numbers to be chosen from $\mathcal{P}_{1}$. Clearly we have $f_{1}\left(n_{1, j+1}\right)=f_{1}\left(n_{1, j}\right)+f_{1}\left(p_{1, j+1}\right)$ and in particular $f_{1}\left(n_{1,1}\right)=f_{1}\left(n_{1,0}\right)+f_{1}\left(p_{1,1}\right)$. Consider the interval

$$
I_{1,0}=\left(\tau_{1,0}-\tau_{1,0}^{1+\xi}-2\left(\tau_{1,0}-\tau_{1,0}^{1+\xi}\right)^{1+\xi}, \tau_{1,0}-\tau_{1,0}^{1+\xi}\right] .
$$

Since

$$
\begin{gathered}
\tau_{1,0}-\tau_{1,0}^{1+\xi}-\left(\tau_{1,0}-\tau_{1,0}^{1+\xi}\right)^{1+\xi}-\left(\tau_{1,0}-\tau_{1,0}^{1+\xi}-\left(\tau_{1,0}-\tau_{1,0}^{1+\xi}\right)^{1+\xi}\right)^{1+\xi} \\
>\tau_{1,0}-\tau_{1,0}^{1+\xi}-2\left(\tau_{1,0}-\tau_{1,0}^{1+\xi}\right)^{1+\xi}
\end{gathered}
$$

$I_{1,0}$ contains an interval of form $\left(v_{j+1}, v_{j}\right]$ with $v_{j+1}=v_{j}-v_{j}^{1+\xi}$. Therefore, we can find $p_{1,1}$ in $\mathcal{P}_{1}$ satisfying

$$
\tau_{1,0}-3 \tau_{1,0}^{1+\xi}<\tau_{1,0}-\tau_{1,0}^{1+\xi}-2\left(\tau_{1,0}-\tau_{1,0}^{1+\xi}\right)^{1+\xi}<f_{1}\left(p_{1,1}\right) \leq \tau_{1,0}-\tau_{1,0}^{1+\xi} .
$$

Let $\tau_{1,1}=\gamma_{1}-f_{1}\left(n_{1,1}\right)=\gamma_{1}-f_{1}\left(n_{1,0}\right)-f_{1}\left(p_{1,1}\right)=\tau_{1,0}-f_{1}\left(p_{1,1}\right)$ so that

$$
\tau_{1,0}^{1+\xi} \leq \tau_{1,1}<3 \tau_{1,0}^{1+\xi}
$$

Inductively we can find prime numbers $p_{1,1}, p_{1,2}, \ldots$ in $\mathcal{P}_{1}$ such that $\tau_{1, j}=\gamma_{1}-$ $f_{1}\left(n_{1, j}\right)$ and

$$
\tau_{1, j-1}^{1+\xi} \leq \tau_{1, j}<3 \tau_{1, j-1}^{1+\xi}
$$

for $j \geq 1$. Since $\tau_{1,0}<\eta<6^{-1 / \xi}$, the intervals $\left[\tau_{1, j}-3 \tau_{1, j}^{1+\xi}, \tau_{1, j}-\tau_{1, j}^{1+\xi}\right]$ are disjoint. Consequently, the prime numbers $p_{1,1}, p_{1,2}, \ldots$ that are chosen at each step from $\mathcal{P}_{1}$ are distinct. By iterating the inequalities we also have

$$
\tau_{1,0}^{(1+\xi)^{j}} \leq \tau_{1, j} \leq 3^{j} \tau_{1,0}^{(1+\xi)^{j}}
$$

for any $j \geq 0$. Moreover,

$$
\tau_{1, j-1}-3 \tau_{1, j-1}^{1+\xi}<f_{1}\left(p_{1, j}\right) \leq \frac{C\left(f_{1}\right)}{p_{1, j}^{\delta}}
$$


and it follows that

$$
p_{1, j} \leq\left(\frac{C\left(f_{1}\right)}{\tau_{1, j-1}-3 \tau_{1, j-1}^{1+\xi}}\right)^{\frac{1}{\delta}} \leq\left(\frac{2 C\left(f_{1}\right)}{\tau_{1, j-1}}\right)^{\frac{1}{\delta}}
$$

for any $j \geq 1$. It follows that for any $j \geq 1$,

$$
n_{1, j} \leq\left(\frac{\left(2 C\left(f_{1}\right)\right)^{\frac{j}{\delta}}}{\left(\prod_{s=0}^{j-1} \tau_{1, s}\right)^{\frac{1}{\delta}}}\right) n_{1,0}
$$

Using the fact that

$$
\prod_{s=0}^{j-1} \tau_{1, s} \geq \tau_{1,0}^{\sum_{s=0}^{j-1}(1+\xi)^{s}} \geq \tau_{1,0}^{(1+\xi)^{j} \sum_{s=1}^{\infty} \frac{1}{(1+\xi)^{s}}} \geq\left(\frac{\eta}{2}\right)^{\frac{(1+\xi)^{j}}{\xi}}
$$

we obtain

$$
n_{1, j} \leq\left(\frac{\left(2 C\left(f_{1}\right)\right)^{\frac{j}{\delta}}}{\left(\frac{\eta}{2}\right)^{\frac{(1+\xi)^{j}}{\xi \delta}}}\right) n_{1,0}
$$

for any $j \geq 0$.

We construct $n_{i, j}$ for $2 \leq i \leq k, j \geq 1$ in a similar manner. More precisely, $n_{i, j}=n_{i, j-1} p_{i, j}$ and the $p_{i, j}$ 's are distinct primes in $\mathcal{P}_{i}$ for each $j \geq 1$. Conditions (i) and (ii) are immediate. Condition (iii) follows from

$$
0<\gamma_{i}-f_{i}\left(n_{i, j}\right) \leq 3^{j} \tau_{i, 0}^{(1+\xi)^{j}} \leq 3^{j} \eta^{(1+\xi)^{j}}
$$

and (2.8) follows from (2.9).

\section{Proof of Theorems 1 And 2}

Suppose $0<\xi^{\prime}<\xi<\lambda / A$ and $v_{0}$ is sufficiently small. Put $L=\left(2 k ! b_{1} \cdots b_{k}\right)^{2}$, let $K$ be the largest prime factor of $L$ and define $\gamma_{j}=\alpha_{j}-f_{j}\left(b_{j}\right)$ for $1 \leq j \leq k$. $\eta$ satisfies (2.7) and also

$$
(\eta / 2)^{\xi^{\prime}}>\eta^{\xi}
$$

Let $n_{i, j}(1 \leq i \leq k, j \geq 0)$ be the sequences of integers guaranteed by Lemma 2 . 
If $k=1$ and $a_{1} \in\{1,2\}$ in Theorem 1 , then $A=1$, each $n_{i, j}$ is odd and for large enough $j$,

$$
\begin{aligned}
\left|f_{1}\left(b_{1}\left(n_{1, j}-1\right)+b_{1}\right)-\alpha_{1}\right| & =\left|f_{1}\left(b_{1} n_{1, j}\right)-\alpha_{1}\right| \\
& \leq \eta^{(1+\xi)^{j}} 3^{j} \\
& =3^{j}\left(\frac{\eta}{2}\right)^{(1+\xi)^{j} \xi^{\prime} / \xi}\left[\eta\left(\frac{2}{\eta}\right)^{\xi^{\prime} / \xi}\right]^{(1+\xi)^{j}} \\
& \leq\left(\frac{\eta}{2}\right)^{(1+\xi)^{j} \xi^{\prime} / \xi}(2 C)^{-j \xi^{\prime}} n_{1,0}^{-\xi^{\prime} \delta} \\
& \leq n_{i, j}^{-\xi^{\prime} \delta}
\end{aligned}
$$

by (3.1). Theorem 1 follows by taking $\xi, \xi^{\prime}$ so that $c<\xi^{\prime} \delta$. The above argument fails when $a_{1}>2$ because we cannot guarantee that infinitely many numbers $n_{1, j}$ are congruent to 1 modulo $a_{1}$, although this can be done in some cases, e.g. $f_{1}(n)=\log (n / \phi(n))$.

When $k \geq 2$ or when $k=1$ and $a_{1}>2$ in Theorem 1 , take $j$ large and consider the system of congruences

$$
\begin{aligned}
& m \equiv 0 \quad(\bmod L) \\
& a_{1} m+b_{1} \equiv 0 \quad\left(\bmod n_{1, j}\right) \\
& a_{2} m+b_{2} \equiv 0 \quad\left(\bmod n_{2, j}\right) \\
& a_{k} m+b_{k} \equiv 0 \quad\left(\bmod n_{k, j}\right) .
\end{aligned}
$$

By the Chinese remainder theorem, this system is equivalent to a single congruence $m \equiv h_{j}\left(\bmod N_{j}\right)$, where

$$
N_{j}=L \prod_{i=1}^{k} n_{i, j}
$$

and $0 \leq h_{j}<N_{j}$. We show that there is a solution $m$ to the above system of congruences such that all the prime factors of

$$
M=\prod_{i=1}^{k} \frac{a_{i} m+b_{i}}{b_{i} n_{i, j}}
$$

are large. This is accomplished with a lower bound sieve. We use the following theorem of Diamond, Halberstam and Richert ([5], [6]).

Theorem DHR. Let $\mathcal{A}$ be a finite set of positive integers, $\mathcal{P}$ a set of primes and let $S(\mathcal{A}, \mathcal{P})$ be the number of integers in $\mathcal{A}$ not divisible by any prime in $\mathcal{P}$. Let $P(z)$ be the product of the primes in $\mathcal{P}$ which are $\leq z$. For real $\kappa \geq 1$, there 
is a continuous, increasing function $f_{\kappa}$ so that if $X \geq y \geq z \geq 2$ and $\omega$ is a multiplicative function satisfying $0 \leq \omega(p)<p$ for $p \in \mathcal{P}, \omega(p)=0$ for $p \notin \mathcal{P}$ and

$$
\prod_{v \leq p<w}\left(1-\frac{\omega(p)}{p}\right)^{-1} \leq\left(\frac{\log w}{\log v}\right)^{\kappa}\left(1+\frac{A}{\log v}\right) \quad(2 \leq v \leq w)
$$

then

$$
\begin{gathered}
S(\mathcal{A}, \mathcal{P}) \geq X \prod_{\substack{p \in \mathcal{P} \\
p \leq z}}\left(1-\frac{\omega(p)}{p}\right)\left(f_{\kappa}\left(\frac{\log y}{\log z}\right)+O_{\kappa, A}\left(\frac{\log \log y}{(\log y)^{1 /(2 \kappa+2)}}\right)\right) \\
-\sum_{\substack{d \mid P(z) \\
d<y}}\left(1+4^{\nu(d)}\right)\left|r_{d}\right|,
\end{gathered}
$$

where $\nu(d)$ is the number of prime factors of $d$ and

$$
r_{d}=\#\{n \in \mathcal{A}: d \mid n\}-\frac{\omega(d)}{d} X
$$

Here the constant implied by the $O$-symbol depends on $\kappa$ and $A$ only. Moreover, $f_{\kappa}(u)>0$ for $u>\beta_{\kappa}$, where $\beta_{\kappa}$ is a certain constant (see e.g. Appendix III of [5]). In particular, $\beta_{1}=2, \beta_{2}<4.2665$ and $\beta_{k}=O(k)$.

To apply the theorem, we take $\kappa=k, \mathcal{P}$ the set of all primes $\leq z$, and

$$
\mathcal{A}=\left\{P(s): 1 \leq s \leq N_{j}^{\mu}\right\}
$$

where

$$
P(s)=\prod_{i=1}^{k} \frac{a_{i}\left(s N_{j}+h_{j}\right)+b_{i}}{b_{i} n_{i, j}}
$$

and $\mu$ is a positive constant. Take $X=N_{j}^{\mu}$ and

$$
\omega(d)=\#\{0 \leq s \leq d-1: P(s) \equiv 0 \quad(\bmod d)\} .
$$

Then $\omega(p)=0$ for $p \mid L$, and by $(1.2), \omega(p) \leq k$ for other $p$. Thus, by Mertens' estimates, (3.2) holds with $\kappa=k$ and $A$ some constant depending only on $k$. Let $\varepsilon>0$ and $y=X^{1-2 \varepsilon}$. Since

$$
4^{\nu(d)}\left|r_{d}\right| \leq 4^{\nu(d)} \omega(d) \leq(4 k)^{\nu(d)} \ll_{\varepsilon} d^{\varepsilon},
$$

we find that

$$
\sum_{\substack{d \mid P(z) \\ d<y}}\left(1+4^{\nu(d)}\right)\left|r_{d}\right| \ll X^{1-\varepsilon}
$$

Take $z=y^{\frac{1-\varepsilon}{\beta_{k}}}=N_{j}^{c_{0}}, c_{0}=\frac{\mu(1-2 \varepsilon)(1-\varepsilon)}{\beta_{k}}$. We find that for large $j$

$$
S(\mathcal{A}, \mathcal{P}) \gg_{k, \mu, \varepsilon} \frac{N_{j}^{\mu}}{\left(\log N_{j}\right)^{k}},
$$


Thus, there is an integer $m \leq N_{j}^{1+\mu}+h_{j}$ such that $m \equiv 0(\bmod L), a_{i} m+b_{i} \equiv 0$ $\left(\bmod n_{i, j}\right)$ for $1 \leq i \leq k$ and all prime factors of

$$
M=\prod_{i=1}^{k} \frac{a_{i} m+b_{i}}{b_{i} n_{i, j}}
$$

are $>z$. There are at most $\left\lfloor\frac{1+\mu}{c_{0}}+1\right\rfloor$ prime factors of $a_{i} m+b_{i}$ which are $>z$. By (b), for $1 \leq i \leq k$ we have

$$
\left|f_{i}\left(a_{i} m+b_{i}\right)-\alpha_{i}\right| \leq\left|f\left(b_{i} n_{i, j}\right)-\alpha_{i}\right|+\left|f\left(\frac{a_{i} m+b_{i}}{b_{i} n_{i, j}}\right)\right| \leq 3^{j} \eta^{(1+\xi)^{j}}+c_{1} N_{j}^{-\delta c_{0}},
$$

where $c_{1}=\left(\frac{1+\mu}{c_{0}}+1\right) \max _{1 \leq i \leq k} C\left(f_{i}\right)$. Moreover, by (2.8) and (3.1), for large $j$ we have

$$
\begin{aligned}
N_{j} & =L \prod_{i=1}^{k} n_{i, j} \leq L\left(2 \max _{1 \leq i \leq k} C\left(f_{i}\right)\right)^{k j / \delta}(\eta / 2)^{-\frac{k(1+\xi)^{j}}{\delta \xi}} \prod_{i=1}^{k} n_{i, 0} \\
& \leq 3^{-k j /\left(\delta \xi^{\prime}\right)} \eta^{-\frac{k(1+\xi)^{j}}{\delta \xi^{\prime}}} .
\end{aligned}
$$

We conclude that for large $j$,

$$
\begin{aligned}
\left|f_{i}\left(a_{i} m+b_{i}\right)-\alpha_{i}\right| & \leq N_{j}^{-\delta \xi^{\prime} / k}+c_{1} N_{j}^{-\delta c_{0}} \\
& \ll m^{-\frac{\delta \xi^{\prime}}{k(1+\mu)}}+m^{-\frac{\delta \mu(1-3 \varepsilon)}{\beta_{k}(1+\mu)}} .
\end{aligned}
$$

Taking $\mu=\frac{\xi^{\prime} \beta_{k}}{k(1-3 \varepsilon)}$ gives

$$
\left|f_{i}\left(a_{i} m+b_{i}\right)-\alpha_{i}\right| \ll m^{-c_{2}} \quad(1 \leq i \leq k),
$$

where

$$
c_{2}=\frac{\delta \xi^{\prime}}{k+\xi^{\prime} \beta_{k}(1-3 \varepsilon)^{-1}} .
$$

Theorem 1 follows by taking $\varepsilon$ sufficiently small and $\xi^{\prime}$ sufficiently close to $\lambda / A$, so that $c_{2}>c$.

Proof of Theorem 2. Without loss of generality, we may assume that $b_{i}>0$ for all $i$. Let $L=\left(2 k ! a_{0} b_{0} \cdots a_{k} b_{k}\right)^{2}$. By (a), there is a number $n_{0}$ with $\left(n_{0}, L\right)=1$ and

$$
f_{0}\left(b_{0} n_{0}\right)>\sum_{i=1}^{k}\left|\zeta_{i}\right|+\max _{1 \leq i \leq k}\left|f_{i}\left(b_{i}\right)\right| .
$$

Let $\alpha_{0}=f_{0}\left(n_{0} b_{0}\right), \alpha_{i}=\zeta_{i}+\alpha_{i-1}$ and $\gamma_{i}=\alpha_{i}-f_{i}\left(b_{i}\right)$ for $1 \leq i \leq k$. Then $\gamma_{i}>0$ for $1 \leq i \leq k$. Let $0<\xi<\xi^{\prime}<\lambda / A$, $v_{0}$ be sufficiently small such that

$$
v_{0}<\min _{p \mid n_{0}, f_{0}(p)>0} f_{0}(p),
$$


and suppose $\eta$ satisfies (2.7) and (3.1). Let $K$ be the largest prime factor of $L n_{0}$, and let $n_{i, j}$ be as in Lemma 2. Consider the system

$$
\begin{array}{rlrl}
m & \equiv 0 & & (\bmod L) \\
a_{0} m+b_{0} \equiv 0 & & \left(\bmod n_{0}\right) \\
a_{1} m+b_{1} & \equiv 0 & & \left(\bmod n_{1, j}\right) \\
& \cdots & & \\
a_{k} m+b_{k} & \equiv 0 & & \left(\bmod n_{k, j}\right)
\end{array}
$$

which is equivalent to a single congruence $m \equiv h_{j}\left(\bmod N_{j}\right)$, where $N_{j}=L n_{0} n_{1, j} \cdots n_{k, j}$ and $0 \leq h_{j}<N_{j}$. Write $m=h_{j}+s N_{j}$.

If $k \geq 2$, we apply Theorem DHR with

$$
\mathcal{A}=\left\{P(s): 1 \leq s \leq N_{j}^{\mu}\right\}
$$

where

$$
P(s)=\frac{a_{0}\left(s N_{j}+h_{j}\right)+b_{0}}{b_{0} n_{0}} \prod_{i=1}^{k} \frac{a_{i}\left(s N_{j}+h_{j}\right)+b_{i}}{b_{i} n_{i, j}},
$$

and $\mathcal{P}$ is the set of primes $\leq z$. Take $X=N_{j}^{\mu}, y=X^{1-2 \varepsilon}$ and $z=y^{\frac{1-\varepsilon}{\beta_{k+1}}}$. The remaining argument is nearly identical to that in the proof of Theorem 1 . The only differences are that $N_{j}$ is a factor $n_{0}$ larger than before, $\kappa=k+1$, we take $\mu=\frac{\xi^{\prime} \beta_{k+1}}{k(1-3 \varepsilon)}$, and

$$
\left|f_{0}\left(a_{0} m+b_{0}\right)-\alpha_{0}\right|=\left|f_{0}\left(\frac{a_{0} m+b_{0}}{b_{0} n_{0}}\right)\right| \ll z^{-\delta} .
$$

We find that

$$
\left|f_{i}\left(a_{i} m+b_{i}\right)-\alpha_{i}\right| \ll m^{-c_{2}} \quad(0 \leq i \leq k)
$$

where

$$
c_{2}=\frac{\delta \xi^{\prime}}{k+\xi^{\prime} \beta_{k+1}(1-3 \varepsilon)^{-1}} .
$$

Taking $\varepsilon$ sufficiently small and $\xi^{\prime}$ sufficiently close to $\lambda / A$ completes the proof.

If $k=1$, we set up the sieve procedure differently. Let

$$
q=\frac{a_{1} L n_{0}}{b_{1}}, \quad r=\frac{a_{1} h_{j}+b_{1}}{b_{1} n_{1, j}} .
$$

We will restrict our attention to numbers $m$ so that $\frac{a_{1} m+b_{1}}{b_{1} n_{1, j}}=q s+r$ is prime. Apply Theorem DHR with

$$
\mathcal{A}=\left\{\frac{a_{0}\left(h_{j}+L n_{0} n_{1, j} s\right)+b_{0}}{b_{0} n_{0}}: N_{j}^{\mu}<s \leq 2 N_{j}^{\mu}, q s+r \text { is prime }\right\}
$$


and $\mathcal{P}$ is the set of primes $\leq z$. Take $X=\frac{1}{\phi(q)}\left(\operatorname{li}\left(2 q N_{j}^{\mu}+r\right)-\operatorname{li}\left(q N_{j}^{\mu}+r\right)\right)$, $y=X^{1 / 2-\varepsilon}$ and $z=y^{(1-\varepsilon) / \beta_{1}}=y^{(1-\varepsilon) / 2}$ for some small fixed $\varepsilon>0$. Here

$$
\operatorname{li}(x)=\int_{2}^{x} \frac{d t}{\log t} .
$$

Each set $\{w \in \mathcal{A}: d \mid w\}$ is either empty, and we take $\omega(d)=0$, or counts primes in a single progression modulo $q d$ which are between $q N_{j}^{\mu}+r$ and $2 q N_{j}^{\mu}+r$, in which case we take $\omega(d)=\phi(q) / \phi(q d)$. Then (3.2) holds with $\kappa=1$ and some absolute constant A. The Bombieri-Vinogradov theorem (e.g. Ch. 28 of [4]) implies that

$$
\sum_{d \leq y}\left(1+4^{\omega(d)}\right)\left|r_{d}\right| \ll \frac{X}{\log ^{5} X} .
$$

Therefore, by Theorem DHR, if $j$ is large then there is a number $s, N_{j}^{\mu}<s \leq 2 N_{j}^{\mu}$ with $q s+r$ prime and all prime factors of $\frac{a_{0}\left(h_{j}+L n_{0} n_{1, j} s\right)+b_{0}}{b_{0} n_{0}}$ are $>z$. For $m=$ $h_{j}+L n_{0} n_{1, j} s$, we therefore have by (b),

$$
\begin{aligned}
\left|f_{0}\left(a_{0} m+b_{0}\right)-\alpha_{0}\right| & =\left|f_{0}\left(\frac{a_{0}\left(m_{0}+L n_{0} n_{1, j} s\right)+b_{0}}{n_{0} b_{0}}\right)\right| \\
& \ll z^{-\delta} \ll N_{j}^{-\delta \mu(1 / 2-\varepsilon)(1-\varepsilon) / 2} \log N_{j}
\end{aligned}
$$

and

$$
\left|f_{1}\left(a_{1} m+b_{1}\right)-\alpha_{1}\right|=\left|f_{1}\left(b_{1} n_{1, j}(q s+r)\right)-\alpha_{1}\right| \ll N_{j}^{-\delta \xi^{\prime}}+N_{j}^{-\mu \delta} .
$$

Hence

$$
\left|f_{1}\left(a_{1} m+b_{1}\right)-f_{0}\left(a_{0} m+b_{0}\right)-\zeta_{1}\right| \ll m^{-\frac{\delta \xi^{\prime}}{1+\mu}}+m^{-\frac{\delta \mu(1 / 2-\varepsilon)(1-\varepsilon)}{2(1+\mu)}} \log m .
$$

Taking $\mu=\frac{2 \xi^{\prime}}{(1 / 2-\varepsilon)(1-\varepsilon)}, \xi^{\prime}$ close enough to $\lambda$ and $\varepsilon$ small enough completes the proof. Finally, if we assume the Elliott-Halberstam conjecture, (3.3) holds with $y=X^{1-\varepsilon}$ and we have, for any $c<\frac{\delta \lambda}{1+2 \lambda}$, that the inequality

$$
\left|f_{1}\left(a_{1} m+b_{1}\right)-f_{0}\left(a_{0} m+b_{0}\right)-\zeta_{1}\right| \ll m^{-c}
$$

holds for infinitely many $m$.

\section{Dealing With polynomial arguments}

Let $f(n)=\log (n / \phi(n))$ or $f(n)=\log (\sigma(n) / n)$. We have $f(p)=1 / p+O\left(1 / p^{2}\right)$, so (b) holds with $\delta=1$. Let $0<\xi<\lambda<1-\Gamma^{\prime}$. We have

(a') $\sum_{p \equiv 1(\bmod 4)} f(p)=\infty$

(c') If $t_{0}$ is small enough, then for any $0<t \leq t_{0}$, there is a prime $p \equiv 1(\bmod 4)$ so that $t-t^{1+\lambda} \leq f(p) \leq t$.

We follow the proof of Theorem 2 (in the case $k \geq 2$ ). Let $n_{0}$ be the product of primes $\equiv 1,3(\bmod 8)$ and such that $f\left(n_{0}\right)>|\zeta|+1$, put $\alpha_{0}=f\left(2 n_{0}\right)$ and 
$\alpha_{1}=\zeta+\alpha_{0}$. Armed with (a') and (c'), an analog of Lemma 1 holds with $k=1$ and $\mathcal{P}_{1}$ consisting only of primes $\equiv 1(\bmod 4)$, and an analog of Lemma 2 holds with the additional restriction that $n_{1, j}$ is the product of only primes $\equiv 1(\bmod 4)$. By our construction of $n_{0}$ and $n_{1, j}$, the system of congruences

$$
\begin{aligned}
m & \equiv 0 & & (\bmod 2) \\
m^{2}+2 & \equiv 0 & & \left(\bmod n_{0}\right) \\
m^{2}+1 & \equiv 0 & & \left(\bmod n_{1, j}\right)
\end{aligned}
$$

has at least one solution $m \equiv h_{j}\left(\bmod N_{j}\right)$ with $N_{j}=2 n_{0} n_{1, j}$ and $0 \leq h_{j}<N_{j}$. Apply Theorem DHR with

$$
\mathcal{A}=\left\{\frac{m^{2}+2}{2 n_{0}} \cdot \frac{m^{2}+1}{n_{1, j}}: m=h_{j}+N_{j} s, 1 \leq s \leq N_{j}^{\mu}\right\}
$$

$\mathcal{P}$ the set of primes $\leq z, X=N_{j}^{\mu}, \kappa=2$ (since $m^{2}+1$ and $m^{2}+2$ are irreducible and coprime, $\omega(p)=2$ on average), $y=X^{1-2 \varepsilon}, z=y^{\frac{1-\varepsilon}{\beta_{2}}}$. There is an $m \leq N_{j}^{1+\mu}+N_{j}$ so that the above system of congruences holds, and all prime factors of $\frac{m^{2}+2}{2 n_{0}} \cdot \frac{m^{2}+1}{n_{1, j}}$ are $>z$. The rest of the argument is the same as in the proof of Theorem 2 .

For the general problem of simultaneously approximating $f_{i}\left(g_{i}(n)\right)$ for $1 \leq i \leq k$, each $g_{i}$ needs to satisfy a version of (a') and (c') where the primes are restricted to those for which $g_{i}(n) \equiv 0(\bmod p)$ has a solution. Also, each $g_{i}$ should have an irreducible factor $g_{i}^{*}$ not dividing any other $g_{j}$. This way, the quantities $f_{i}\left(g_{i}^{*}(n)\right)$ will be sufficiently independent to allow the method to work. Analogous to Theorem 1 , with appropriate restrictions on $\alpha_{i}$, the system

$$
\left|f_{i}\left(g_{i}(n)\right)-\alpha_{i}\right|<n^{-c} \quad(1 \leq i \leq k)
$$

will have infinitely many solutions for some $c>0$. Here $c$ will depend on $\delta, A, k$ and the number of irreducible factors of each $g_{i}$ and each $\left(g_{i}, g_{j}\right), i \neq j$. Similarly, for any $\zeta_{1}, \ldots, \zeta_{k}$ the system

$$
\left|f_{i}\left(g_{i}(n)\right)-f_{i-1}\left(g_{i-1}(n)\right)-\zeta_{i}\right|<n^{-c} \quad(1 \leq i \leq k)
$$

will have infinitely many solutions for some $c>0$.

\section{REFERENCES}

[1] E. Alkan, G. Harman and A. Zaharescu, Diophantine approximation with mild divisibility constraints, J. Number Theory 118 (2006), 1-14.

[2] R. C. Baker, Diophantine Inequalities, London Math. Soc. Monogr. (N.S.), vol. 1, Oxford Univ. Press, New York, 1986.

[3] R. C. Baker, G. Harman and J. Pintz, The difference between consecutive primes. II., Proc. London Math. Soc. (3) 83 (2001), no. 3, 532-562. 
[4] H. Davenport, Multiplicative number theory, 3rd ed., Graduate Texts in Mathematics vol. 74, Springer-Verlag, New York, 2000.

[5] H.Diamond, H. Halberstam and H. -E. Richert, Combinatorial sieves of dimension exceeding one, J. Number Theory 28 (1988), 306-346.

[6] H.Diamond, H. Halberstam and H. -E. Richert, Combinatorial sieves of dimension exceeding one II, In Analytic Number Theory, Proceedings of a Conference in Honor of Heini Halberstam, 265-308 (B.C. Berndt, H. G. Diamond and A. J. Hildebrand, eds.). Birkhäuser (1996).

[7] P. Erdős, Some remarks on Euler's ф function, Acta Arith. 4 (1958), 10-19.

[8] P. Erdős and A. Schinzel, Distributions of the values of some arithmetical functions, Acta Arith. 6, (1960/61), 473-485.

[9] P. Erdős and A. Wintner, Additive arithmetical functions and statistical independence, Amer. J. Math. 61 (1939), 713-721.

[10] J. B. Friedlander, Fractional parts of sequences, Théorie des nombres (Quebec, PQ, 1987), 220-226, de Gruyter, Berline, 1989.

[11] S. W. Graham, J. J. Holt and C. Pomerance, On the solutions to $\phi(n)=\phi(n+k)$, Number theory in progress, Vol. 2 (Zakopane-Kościelisko, 1997), 867-882, de Gruyter, Berlin, 1999.

[12] H. Halberstam, H.-E. Richert, Sieve methods, LMS Monographs No. 4, Academic Press, London (1974).

[13] G. Harman, A. Kumchev, and P. A. Lewis, The distribution of prime ideals of imaginary quadratic fields, Trans. Amer. Math. Soc. 356 (2004), no. 2, 599-620.

[14] F. Luca and I. E. Shparlinski, Approximating positive reals by ratios of kernels of consecutive integers, Diophantine analysis and related fields 2006, 141-149, Sem. Math. Sci., 35, Keio Univ., Yokohama, 2006.

[15] A. Schinzel, On functions $\phi(n)$ and $\sigma(n)$, Bull. Acad. Pol. Sci. Cl. III 3 (1955), 415-419.

[16] I. Schoenberg, Über die asymptotische Verteilung reeller Zahlen mod 1, Mat. Z. 28 (1928), 171-199.

[17] D. Wolke, Eine Bemerkung über die Werte der Funktion $\sigma(n)$, Monatsh. Math. 83 (1977), no. 2, 163-166. (German. English summary)

Department of Mathematics, Koc University, Rumelifeneri Yolu, 34450, Sariyer, Istanbul, TURKEY.

e-mail: ealkan@ku.edu.tr

Department of Mathematics, University of Illinois at Urbana-Champaign, 1409 W. Green Street, Urbana, IL, 61801, USA.

e-mail: ford@math.uiuc.edu

e-mail: zaharesc@math.uiuc.edu 